\title{
Woman Security using Android based Hybrid Framework
}

\author{
Sukhjeen Kaur \\ M.Tech, CSE, \\ Department of computer science \\ Punjabi University, Patiala \\ Punjab, India
}

\author{
Jagpuneet Kaur Bajwa \\ Assistant Prof. \\ Department of Computer Science \\ Punjabi University, Patiala \\ Punjab, India
}

\begin{abstract}
In today's world, the necessary question that is essential to arise in every one's mind is regarding the security of their beloved ones, mostly their daughters, wives, friends, sisters, cousins. As the issues on women molestation is increasing day by day. Even every woman is also worried about her own security and safety, as she cannot walk freely in odd hours. This paper provide an Android based Smart phone application which help the woman during the problem for instant services from nearest police station or NGO based on instant message ,Gps based correct location detection using Google map with in specified diameter.
\end{abstract}

\section{Keywords}

Instant message

\section{INTRODUCTION}

The prestige of women have been facing many challenges from the past few millennium. In Indian history many of the social workers did fight for the women's rights. Women were never teach how to stand independently. She did not get an equal status to men, whether it's about their rights, physically strong, socially or economically. Women are considered safe when they act to the will of men, And are unsafe when they act according to their own wish. In today's world, women have got equal rights in almost all fields, they work as pilots, politicians, scientists, engineers, defense services. Women have captured political positions i.e. Prime Minister, speaker, President. women have also achieved in administration, judicial and executive services.

In spite of all the social reforms of women, even then woman face many social challenges, i.e. violence, harassment, victim of abuse, molestation. This paper focuses on security, safety, and protection of women. When women feel insecure she can use this application for her safety. The system consists of various modules such as GSM shield, GPS, screaming alarm, set of pressure sensors for activation. The Delhi Nirbhaya case has shocked up the whole world and it was the greatest motivation for this system. It was the high time we women need the change. Finally, in the last section results will be discussed which are obtained from the system and conclusion will be made on the basis of result obtained.

\section{ANDROID OPERATING SYSTEM}

In today's world, Android rules the Smartphone's universe, which is truly an open source. It is a mobile platform that bring transformation in the android applications by using advanced level of hardware and software. Android platform has the latest security architecture that provides the ensured security to applications, information, user data and network. Android is designed with multilayered security that provides flexibleness needed for an open platform, whereas providing protection for all users of the platform designed to a software stack, android includes an operating system, middleware and core application as a complete. Android powers millions of mobile devices in more than 190 countries around the world. Android is most commonly used operating system. According to the exploration done in April 2013, 71\% of android phones were in use and In November 2013, android share global Smartphone led by Samsung, Its popularity became $81 \%$. Android operating system provides unique and extendable features like additional hardware support, video calling, updated user interface design. Most of the android applications available on Google play are free of cost. Android is also designed with focused on user's perspective. Users can view how applications work, and manage those applications.

\section{DRAWBACKS OF EXISTING APP's 3.1 VithU Application}

VithU, is an emergency App that sends alert messages at every 2 minutes to your registered contacts by consecutively pressing the power button of your phone.

The message says "I am in danger. I need help. Please follow my location." The receiver will be updated with your current location and application will update your location after every 2 minutes. Whereas user will get the safety tips by pressing "Tips Feed" option exclusively in an emergency situation. This application is developed by "Channel V" under the "Gumrah" initiatives. Application also reminds the user whenever "Gumrah" is on air and it provides an option to post one's own incident with Channel V. Drawbacks of the application-1) Needs compulsory internet connection.

2) Balance is must in the phone to send messages.

3) App has not been optimized for the low-to-mid range phones.

Rating of this application is 4.2 / 5 .

\subsection{Nirbhya application}

NIRBHAYA is an android application that sends a call or messages to the nominated contacts or group. Correctness and communication of the application depends upon the hardware/software and active data plan, SMS plan, talk time and active GPS functionality. Features in Nirbhaya: Be Fearless :

1. Geo fence: User can create Geo fence at insecure places, so that when the user enter and exit that area Geo fence will inform their guardians.

2. Stamp: User can mark location on map as aa insure or unsafe place, to aware other people about it. 
3. Power button: Pressing power button will send SOS messages.

4. Shake to alert: When user shake the phone, emergency messages will be send to defined contacts even if the phone is locked.

5. Unsafe area alert: User will get an alert when they will enter the stamp marked area.

6. Application alert the defined contacts or group by sending them SMS or through phone call or post the user's location on Facebook using Global positioning System(GPS).

7. Drawbacks- 1) Last update of this app is JUNE 20 , 2014

2) In this App STAMP option is not performing correct work.

Rating of this application is 4.2 / 5 .

\section{3 bSafe Application}

bSafe is a personal safety application that is designed to keep you safer $24 / 7$. It provides safety in real emergencies, making it the ultimate safety tool for everyone you love. bSafe puts safety in YOUR hands - for FREE!

Have you ever felt unsafe when walking alone at night, or jogging on a running trail? Have you ever needed an excuse to get out of a bad date or long meeting, or simply been looking for an easier way to hook up with your closest friends? Have you ever worried about how you actually would get help in case of an emergency?

bSafe can help in situations:

- Create one's own safety network that can include guardians, police, friends.

- User can share his/her current location, by using I'm here.

- With the Follow Me's live GPS trace system, any of your friend or guardian can walk along you from their own location.

- An alarm will be triggered automatically, if user have not checked in at time.

- You can make your phone ring as a fake call so that you can get out of the specific situation. You can also define the fake call that whose call it is.

- When the user is in problem, he/she can press guardian alert button that will inform your list members that you are in trouble and need help. And it will share your location too.

bSafe puts your safety in your hands and allow you to stay connected with your loved ones.

Drawbacks- 1) In this app LOCATION ACCURACY feature is not which is mandatory regarding safety purpose.

2) If person wants to use this app then SUBSCRIPTION IS mandatory.

Rating of this application is 4.1 / 5

\subsection{Hollaback Application}

Hollaback! is an application that help to end the street harassment, which is major issue in the current world. With the use of mobile technology you can step forward to end street harassment.

In Hollaback! application user can share their stories and can warn other people and user can also read the other people stories too.

Drawbacks- 1) In this user can upload stories but these stories are unable to upload properly.

2) This Application works only in major cities and metropolitan areas.

Rating of this application is 3.7 / 5

\subsection{Circle of 6 Application}

Circle of 6 is a personal safety application which is specifically for school and college students. Circle of 6 allows you to select six friends from the campus. when the user is in problem, user can double press the phone screen and then the pre-programmed SMS alert message will be send to the selected six friends along with the user's location. circle of 6 also inform the critical resources and hotlines of the campus that are for the students safety.

Drawbacks- 1) This app don't collect any identify info from user end.

2) In this app AGE requirement that is 18 or older than 18 .

3) This App is only for the college students (only three colleges are there in the list) not for general public.

Rating of this application is 4.5 / 5

\subsection{Fightback application}

Fightback is a safety application, developed by 'Tech Mahindra' for the safety of their employees. It sends SOS alerts from user's phone. This application uses GPRS, GPS, SMS, location and your Facebook account to inform that you are in trouble.

In this application users location information is send to server through GPS(Global Positioning System). Its working is simple, You can download the application and then register yourself. After registration, you can login into the application.

Another feature of this application is that you can search your blood group donors, As the details has to be filled in the application regarding your blood group and then on a single click you can find the donors.

Drawbacks:- 1) In this application whenever user wants to send SMS then it display alert message that is COULD NOT CONNECT TO SERVER.

2) Also this app mostly hangs up.

Rating of this application is $3.8 / 5$

\section{7 iFollow Application}

iFollow is a security application in which you can add three(3) contacts that can help you when you are in problem. You just have to shake your phone for three times in five seconds. Then the application will call the first contact person and if he doesn't respond to the call, application will send an SMS to all three contacts along with your location. On 10 meters of change in your location application will again send SOS messages with new location. 
Drawbacks- 1) This App crashes when user presses EMERGENCY continuously.

2) User can't test without turning on GPS

3) This app is unable to give accurate LOCATION.

Rating of this application is 4.5 / 5

\subsection{Scream Alarm Application}

This application make your mobile phone scream's loudly with a woman's voice by pressing the application's button. The screaming alarm will let to know another people present at the current location that you need help.

Drawbacks- 1) In this app like as mentioned, it give loud volume but in reality it give low. so that's why it's downloading rate is less.

2) Require you to unlock your phone and look for the app in dangerous situations.

Rating of this application is 3.7 .

\subsection{Raksha Application}

Raksha- Woman safety alert application that help women by the press of button. Your location will be shared with your selected contacts and application will alert the contacts with a loud buzzer. Through this application your added contacts can see your current location and you too can get their location by requesting them to share their location with you. Few features of application are:

- When application is not running, you can send SOS by pressing volume key for three seconds.

- Geo fence.

- Booming alarm.

- Danger zone or unsafe area alert.

Drawbacks- 1) Receiver can get or receive message when he/she have same application on his/her device.

Rating of this application is $4.0 / 5$

\section{MOBILE CLOUD COMPUTING}

Currently, people move from traditional way to modern and flexible way of saving, keeping and accessing files as well as applications. People prefer to save and retrieve their files anywhere at any time via any type of devices. That is the reason why cloud computing comes into the picture. By using cloud computing, it could reduce costs especially in maintaining the hardware and software. Besides reducing costs, cloud computing offers other benefits in speeding up network communication, scalability, protection against network attacks, data storage and back-up solutions, on demand and real time detection of system tampering. This research helps in expanding the technology of cloud computing since it is considered as a new but rapidly growth technology. Previously, companies, organizations and institutions used cloud computing in their businesses. As many researchers reported that there are various benefits of using cloud computing such as maximize profits and minimize costs. Now, the use of cloud computing is not among companies, organizations and institutions only but it is used widely by individuals where it could help them to access their files anywhere at any time via any type of devices. For instance, online games can be played by people, provided that he has an Internet access. In 2013, one of the researches reported that there are three main areas in mobile cloud computing that have not been really solved, i.e. working while on the move, security towards cloud and incentive for surrogates. Therefore, it is worth to be investigated and researched in this area since there are various drawbacks to be solved. Cloud computing is a model for on-demand network access. There is a combination of more components such as network resources, storage, servers, applications and services to form a cloud computing. Cloud computing refers to services offered over the Internet and data centers that provide the services. The word cloud computing always refers as Software as a Service (SaaS) and usually it involves applications software. However, the word data center is called as a cloud and it involves hardware and system software. There are six characteristics to form a cloud computing: on-demand self-service, broad network access, resource, pooling, rapid elasticity, measured service.

\section{WHATSAPP}

WhatsApp is a cross-platform mobile messaging application that widely used by mobile users. It is a widely used application to exchange text messages, videos, images, audios, create group chat, and sharing contact numbers. It is popular because this application offers messages to be sent and received at no cost. The communication happens in real time basis, provided Internet connection must be present during that time. An investigation done by Dutch Data Protection Authority showed that all successfully sent messages will not be saved on the WhatsApp server, instead it will be saved on sender's and receiver's smart phones. However, unsent messages will be stored for 30 days on the WhatsApp server and only will be deleted from the server once it is sent. According to Mahajan et.al, it was found that all user activities in WhatsApp were logged into the mobile internal memory with folders and files created to store the activities including date and time stamp. Some of the files such as msgstore.db were identified to store all chat lists and chat messages, wa.db file stores contact number and com. WhatsApplfiles $\backslash$ folder stores pictures of people in the chat list. Even the users are allowed to delete the messages and files from their smart phones, the deleted items were not actually vanished. On the Android smart phones, these items will be stored in backup folder. Meanwhile, iPhone users were given a choice to make backups by synchronizing the files from their iPhone to iTunes or iCloud. On the WhatsApp server side however, will store, delete and remove the delivered files within a short period of time.

\section{TRACKING PEOPLE}

The time, position and velocity of any GPS receiver can be found with the help of GPS. The constellations of 27 earth orbiting satellites make the Global Position System. These 27 earth orbiting satellite includes 24 in operation and 3 extras, in case one fails. This satellite network was invented by the U.S. military for the purpose of the military navigation system but later it was opened to everybody else for general purpose use. These highly solar powered satellites (30004000 pound power) revolve around the globe about 19,300 $\mathrm{km}$ (12,000 miles), completing two rotation of oblate spheroid globe. The arrangement of the satellites on the orbits is such that at any point of time or at anywhere on earth there are minimum four satellites visible in the sky. The function of GPS receiver is to find out the position of four or more satellites and to find out the coordinates of each satellite. With the help of this information it can deduce its own location. This whole operation is called tri-Iateration. 
To find the coordinates of the object, the following things are required by the GPS receiverr: 1) To get at least three satellite's position above site 2) distance between the each of those satellites and the site. This very high frequency as well as low power radio signals analyze from the GPS satellites by GPS receiver. For improving the better performance to pick up signals multiple receivers has been used. To get the detailed map of a place one can connect the GPS receiver by a computer from which complete information can be obtained by receiver's latitude and longitude readouts. Some GPS receivers are capable to download the maps with full description into memory or supply the complete maps with plug-in map cartridges. A GPS receiver is also capable to detect the moment of the object. If the receiver is moving on left side then by using the change in location it can stay in constant communication with GPS satellites. With the help of this information and its built-in clock, the GPS receiver can give the valuable information: - About the distance travelled by the object (odometer) - About the distance travelled by the object - About the current speed of the object (speedometer) - About the Average speed of the object • About the exact location of the object (bread crumb trail show it) - The estimated time of arrival at the destination if the current speed is preserved. If only splitting is used, the final partition may contain adjacent regions with identical properties. This drawback can be remedied by allowing merging as well as splitting i.e. merge any adjacent regions $\mathrm{Rj} \& \mathrm{Rk}$ for which, $\mathrm{Q}(\mathrm{Rj} \mathrm{U} \mathrm{Rk})=$ TRUE. Stop when no further merging is possible.

\section{CONCLUSION AND FUTURE WORK}

Android operating is one of the cheapest and very demanding smart phone right now. It is an open source due to this code of OS can easily customized. Using this Android OS we try to control various parts of phone which help the woman during the problem like shaking the phone will send the message to Ngo's etc. This shaking speed of phone is compute using android function based on that matched condition message send instead send message on normal shaking of phone.

\section{REFERENCES}

[1] Akinode 1.1., Alawode A.J. and Ojuawo 0.0., 2011 "Improving National Security Using GPS Tracking System Technology," Proceedings of the I st International Technology, Education and Environment Conference African Society for Scientific Research (ASSR), Ogun State, Nigeria.

[2] Al-Bayari, 0., B. Sadoun, Vol 18, Issue 9, April 2005 "New centralized automatic vehicle location communications software system under GIS environment," International Journal of Communication Systems.

[3] Car-Animal Accidents Raise Concern By Constance 1. Hays. Published: January 02, 1993.Available: http://www.nytimes.comlI993/0 1/02/nyregion/car -an imal-accidentsraise-concern.html

[4] CGALIES, "Report on Implementation Issues Related to Access to detection Information by emergency Services(E I 12) in the European Union," CGALIES, Final Report.

[5] Chris Thompson, Jules White, Brian Dougherty, Adam Albright and Douglas C.Schmidt, Vanderbilt
University, Nashville, TN USA," Using Smart phones to Detect Car Accidents and Provide Situational Awareness to Emergency Responders".

[6] Coastal Navigation, Global Positioning Systems, Available: http://www.vmrgladstone.org.au/GPS Basics.htm

[7] Datasheets of microcontroller AT89C51, GSM module, 16*2 LCD,Available: http://www.alldatasheet.com

[8] Fan, x., W. Xu, H. Chen, and 1. Liu, Volume 2, Issue 18-20, April 2006 "CCSMOMS:A Composite Communication Scheme for Mobile Object Management System," 20th International Conference on Advanced Infonnation Networking and Applications.

[9] Hapsari, A.T., E.Y. Syamsudin, and I. Pramana, 2005 "Design of Vehicle Position Tracking System Using Short Message Services And Its Implementation on FPGA," Proceedings of the Conference on Asia South Pacific Design Automation, Shanghai, China.

[10] Hsiao, W.C.M., and S.KJ. Chang, 2006 "The Optimal Location Update Strategy of Cellular Network Based Traffic Information System," Intelligent Transportation Systems Conference.

[11] Insurance Issue Institue. "III Presentations", Available: http://www.iii.org/media/hottopics/insurance/test4

[12] Martin Backstrom, Andreas Hardrup, Tomas Nylander, Jari Vikberg and Peter Ohman, Ericsson Review, No.2, 2005 "Mobile Home-GSM Services over Wireless LAN".

[13] Missing children in India, "Child protection and child rights,"Available:http://www.childlineindia.org.in/missi ng-children-india.htm

[14] Pati, N., December 2007 "Occlusion Tolerant Object Recognition Methods for Video Surveillance and Tracking of Moving Civilian Vehicles," MS Thesis (Computer Engineering), University of North Texas, Denton, USA.

[15] Road Accidents in India by Government of India, Ministry of Road Transport and Highways, Transport Research Wing, Delhi, 2010.

[16] Tamil, E.M., D.B. Saleh, and M.Y.I. Idris, May 2007, "A Mobile Vehicle Tracking System with GPS/GSM Technology," Proceedings of the 5th Student Conference on Research and Development (SCORED), Permala Bangi, Malaysia.

[17] UDOT Wildlife and Domestic Animal Accident Toolkit by Utah Department of Transportation Environmental Services \& Wildlife \& Domestic Animal Accident Quality Improvement Team. Report No. UT 08.07,4501 So. 2700 West Salt Lake City, Utah 84114 April 29, 2008.

[18] US Air Force Fact Sheet: Global Positioning Systems Wing, Los Angles Air Force Base, Available: Technologies for computing and telecommunication. 